\title{
ON A TWO-POINT BOUNDARY VALUE PROBLEM FOR SECOND-ORDER DIFFERENTIAL INCLUSIONS ON RIEMANNIAN MANIFOLDS
}

\author{
YURI E. GLIKLIKH AND ANDREI V. OBUKHOVSKII \\ Received 27 August 2002
}

We consider second-order differential inclusions on a Riemannian manifold with lower semicontinuous right-hand sides. Several existence theorems for solutions of two-point boundary value problem are proved to be interpreted as controllability of special mechanical systems with control on nonlinear configuration spaces. As an application, a statement of controllability under extreme values of controlling force is obtained.

\section{Introduction and motivation}

The main object of research in this paper is a mechanical system with set-valued force given in geometrically invariant terms. This language allows us to consider, from unique mathematical point of view, a broad class of real mechanical systems including those on curved nonlinear configuration spaces, forces with control, and so forth. First we introduce some basic notions in order to set up the problem. Details in geometry of manifolds can be found, for example, in $[1,4,5]$, in set-valued analysis-for example, in [2, 6, 7]. Some definitions, used here, can be also found in Section 2.

Let $M$ be a Riemannian manifold. Recall that, this means that in any tangent space $T_{m} M$, the scalar product $\langle\cdot, \cdot\rangle_{m}$ is given and $\langle\cdot, \cdot\rangle_{m}$ is smooth in $m \in M$. The total family of those scalar products is called a Riemannian metric. We will omit the index $m$ in notation of the form when it does not yield a confusion. The norm in the tangent space, generated by the above scalar product, will be denoted by the usual symbol $\|\cdot\|$.

We interpret $M$ as the configuration space of a mechanical system. Tangent vectors to $M$ are interpreted as velocities. The function $\mathscr{K}: T M \rightarrow T M, \mathscr{K}(X)=$ $(1 / 2)\langle X, X\rangle$ on the phase space $T M$ (the tangent bundle to $M$ ) is the kinetic energy of mechanical system. The (nonautonomous) force field $\alpha(t, m, X)$ is a 
1-form on $M$ that at any configuration $m \in M$ may depend on time $t \in \mathbb{R}$ and velocity $X \in T_{m} M$.

We assume the Riemannian manifold $M$ to be complete. The mechanical meaning of this assumption is that a free particle on the configuration space $M$ does not go to infinity in finite time. The Riemannian metric enables us to identify differential 1 -forms and vector fields on $M$, and henceforth, we regard the force field as a vector field, also depending on time and velocity. Denote by $\pi: T M \rightarrow M$ the natural projection, that is, $\pi\left(T_{m} M\right)=m$ for any tangent space $T_{m} M$. Thus, the vector force field can be considered as a map $\alpha: \mathbb{R} \times T M \rightarrow T M$ such that $\pi \alpha(t, m, X)=m$ for all $t \in \mathbb{R}$ and $(m, X) \in T M$.

The equation of motion for the system is Newton's second law in the following geometric form:

$$
\frac{D}{d t} \dot{m}(t)=\alpha(t, m(t), \dot{m}(t))
$$

where $D / d t$ is the covariant derivative of Levi-Civita connection on $M$ and $\alpha$ is the vector force field (see above).

Consider a mechanical system with control. Then at any point $(m, X)$ of phase space and time instant $t$, the set $F(t, m, X) \subset T_{m} M$ of all values of the force determined by all possible values of controlling parameter is given. Thus, the trajectory of such a system satisfies the following differential inclusion:

$$
\frac{D}{d t} \dot{m}(t) \in F(t, m(t), \dot{m}(t))
$$

that is a set-valued version of Newton's law (1.1).

Definition 1.1. A $C^{1}$-curve $m(t)$, such that its derivative is absolutely continuous and inclusion (1.2) holds for $m(t)$ almost everywhere (a.e.), is called a solution of inclusion (1.2).

In this paper, we investigate the two-point boundary value problem for (1.2), that is, the existence of a solution $m(t)$ such that for given points $m_{0}, m_{1} \in M$ and time instants $t_{0}, t_{1}$ the relations $m\left(t_{0}\right)=m_{0}$ and $m_{1}=m_{1}$ hold. If such a trajectory exists, there exists also a curve in the domain of controlling parameter such that using this (time-dependent) control, we can derive the trajectory to $m_{1}$ at $t_{1}$ from $m_{0}$ at $t_{0}$. This means the controllability of the system for given $m_{0}, t_{0}$ and $m_{1}, t_{1}$.

It should be pointed out that the two-point boundary value problem on curved (nonlinear) configuration spaces (unlike that on flat linear spaces) may not be solvable even for single-valued bounded smooth forces. We can mention, for example, examples of systems on two-dimensional sphere from [5] where some or all couples of antipodal points cannot be joint by a trajectory of the system. 
We show (see Theorem 3.1) that a point $m_{1}$ is accessible from $m_{0}$ at least within small enough time interval if $m_{1}$ and $m_{0}$ are not conjugate at least along one geodesic curve on $M$ (notice that antipodal points on two-dimensional sphere are conjugate along all geodesics joining them). We deal with bounded lower semicontinuous (lsc) (or almost lsc, see Definitions 2.4 and 3.7) set-valued forces $F(t, m, X)$ not necessarily having convex images.

This is important for applications because of the following example of setvalued forces of the above-mentioned sort. Consider a set-valued bounded and Hausdorff continuous force $A(t, m, X)$ with convex closed images. Then (see Lemma 3.5) the set-valued force $\operatorname{Ext} A(t, m, X)$, sending $(t, m, X)$ into the set of extreme points of $A(t, m, X)$, is lsc. Obviously, under the above assumptions on $A(t, m, X)$, the force $\operatorname{Ext} A(t, m, X)$ is bounded and may not have convex images. The solvability of the two-point boundary value problem with the force $\operatorname{Ext} A(t, m, X)$ (see Theorem 3.6) means controllability of the system with force $A(t, m, X)$ for given points under extreme values of controlling force. This fact cannot be covered by previous existence theorems for upper semicontinuous forces with convex images (see, e.g., [5]).

The structure of this paper is as follows. In Section 2, we construct some special operators of integral type, based on the use of Riemannian parallel translation, and the so-called velocity hodograph equation that form a geometric machinery for investigating the problem. In this section, we also present some facts from set-valued analysis, applied below. Section 3 is devoted to proving the solvability results for the above problem.

\section{Description of the involved machinery}

Let $M$ be a complete Riemannian manifold. Consider $m_{0} \in M, I=[0, l] \subset \mathbb{R}$, and let $v: I \rightarrow T_{m_{0}} M$ be a continuous curve.

Theorem 2.1 (see $[3,5]$ ). There exists a unique $C^{1}$-curve $\gamma: I \rightarrow M$ such that $\gamma(0)=m_{0}$ and the tangent vector $\dot{\gamma}(t)$ is parallel to the vector $v(t) \in T_{m_{0}} M$ for every $t \in I$.

Indeed, the curve $\gamma$ is represented as $\gamma(t)=\delta^{-1}\left(\int_{0}^{t} v(\tau) d \tau\right)$ where $\delta$ is Cartan's development and $\delta^{-1}$ is its inverse map developing $C^{1}$-curves from $T_{m_{0}} M$ into $M$ (see, e.g., [1] for details).

In what follows, we denote by $\mathscr{S} v(\cdot)$ the curve $\gamma$ constructed as above beginning with $v$.

Consider the Banach space $C^{0}\left(I, T_{m_{0}} M\right)$ of continuous maps from $I$ to $T_{m_{0}} M$ and the Banach manifold $C^{1}(I, M)$ of $C^{1}$-smooth maps from $I$ to $M$. As follows from Theorem 2.1, the operator $\mathscr{S}: C^{0}\left(I, T_{m_{0}} M\right) \rightarrow C^{1}(I, M)$ is well posed. If $M$ is a Euclidean space, then $\mathscr{S} v$ is the primitive of $v$.

The mapping $\mathscr{S}$ is a homeomorphism between $C^{0}\left(I, T_{m_{0}} M\right)$ and its image $C_{m_{0}}^{1}(I, M)$ in $C^{1}(I, M)$, where the manifold $C_{m_{0}}^{1}(I, M)$ consists of all $C^{1}$-curves $\gamma$ with $\gamma(0)=m_{0}$. 
Let $m(t)$, where $t \in I$ and $m(0)=m_{0}$, be a $C^{1}$-curve in $M$ and let $\alpha(t, m, X)$ be a single-valued force field. Denote by $\Gamma \alpha(t, m(t), \dot{m}(t))$ the curve in $T_{m_{0}} M$ such that the vector $\Gamma \alpha(t, m(t), \dot{m}(t))$ is parallel to $\alpha(t, m(t), \dot{m}(t))$ along $m(\cdot)$ for every $t$ (i.e., $\Gamma \alpha(t, m(t), \dot{m}(t))$ is obtained by parallel translation of vectors $\alpha(t, m(t), \dot{m}(t))$ along $\gamma(\cdot)$ at $\left.T_{m_{0}} M\right)$.

Specify a vector $C$ in $T_{m(0)} M$ and consider the integral equation

$$
m(t)=\mathscr{S}\left(\int_{0}^{t} \Gamma \alpha(\tau, m(\tau), \dot{m}(\tau)) d \tau+C\right)
$$

on $I=[0, l]$. It is shown in [3] (see also $[4,5]$ ) that $(2.1)$ is the integral form of the second Newton law (1.1), that is, its solution is the trajectory of mechanical system with force $\alpha$ having the initial conditions $\gamma(0)=m_{0}$ and $\dot{\gamma}(0)=C$.

Let $m(t), t \in I$, be a trajectory of the mechanical system, that is, a solution of (2.1).

Definition 2.2. The velocity hodograph of the trajectory $m(t)$ is the curve $v: I \rightarrow$ $T_{m(0)} M$ such that $v(t)$ is parallel to $\dot{m}(t)$ along $m(\cdot)$.

It is not hard to see that the velocity hodograph of a solution of (2.1) satisfies the equation

$$
v(t)=\int_{0}^{t} \Gamma \alpha\left(\tau, \mathscr{Y} v(\tau), \frac{d}{d \tau} \mathscr{Y} v(\tau)\right) d \tau+C
$$

It is obvious that if $v$ is a solution of (2.2), then $\mathscr{V} v$ is a solution of (2.1), that is, a trajectory of the mechanical system. Below we will reduce the inclusion (1.2) to a certain integral relation similar to the velocity hodograph equation.

Theorem 2.3. Let a point $m_{1} \in M$ be not conjugate with $m_{0}$ along some geodesic of the Levi-Civitá connection on $M$. Then for any geodesic $a(t),\left(a(0)=m_{0}, a(1)=\right.$ $m_{1}$ ) along which $m_{0}$ and $m_{1}$ are not conjugate, and for any number $k>0$, there exist a number $L\left(m_{0}, m_{1}, k, a\right)>0$ such that for $0<t_{1}<L\left(m_{0}, m_{1}, k, a\right)$ and for any curve $u(t) \in U_{k} \subset C^{0}\left(\left[0, t_{1}\right], T_{m_{0}} M\right)$ (where $U_{k}$ is the ball of radius $k$ ), there exists, in a certain bounded neighbourhood of the vector $t_{1}^{-1} a(0) \in T_{m_{0}} M$, a unique vector $C_{u} \in T_{m_{0}} M$ continuously dependent on $u$ such that $S\left(u+C_{u}\right)\left(t_{1}\right)=m_{1}$.

This statement is proved as [3, Theorem 1.3] and [5, Theorem 3.3].

We will also use some facts from multivalued theory. A set-valued map (or a multimap) $F$ from a metric space $\Omega$ into a metric space $X$ is a map sending any point $\omega \in \Omega$ to a nonempty subset $F(\omega) \subset X$.

Definition 2.4. A multimap $F: \Omega \rightarrow X$ is said to be lsc at $\omega_{0} \in \Omega$ if for any $\varepsilon>0$, there exists $\delta>0$ such that for any $\omega$ belonging to $\delta$-neighbourhood of $\omega_{0}$, the set $F\left(\omega_{0}\right)$ is contained in the $\varepsilon$-neighbourhood of $F(\omega)$. The multimap $F$ is called lsc if it is lsc at any $\omega \in \Omega$. 
Definition 2.5. A multimap $F: \Omega \rightarrow X$ is said to be upper semicontinuous at $\omega_{0} \in \Omega$ if for any $\varepsilon>0$, there exists $\delta>0$ such that for any $\omega$ belonging to $\delta$-neighbourhood of $\omega_{0}$, the set $F(\omega)$ is contained in the $\varepsilon$-neighbourhood of $F\left(\omega_{0}\right)$. The multimap $F$ is called upper semicontinuous if it is upper semicontinuous at any $\omega \in \Omega$.

Definition 2.6. A multimap $F: \Omega \rightarrow X$ is called Hausdorff continuous if it is both upper and lsc.

Definition 2.7. A selection $f$ of the set-valued map $F: \Omega \rightarrow X$ is a single-valued map $f: \Omega \rightarrow X$ such that $f(\omega) \in F(\omega)$ for all $\omega \in \Omega$.

We will be interested in existence of continuous selections of set-valued maps. Notice that if $F$ is a lsc set-valued map of Banach spaces with closed convex images, by famous Michael's theorem, then it has a continuous selection; but it is not the case if either $F$ is not lsc or has not convex images.

Definition 2.8. Let $E$ be a separable Banach space. A nonempty subset $M \subset$ $L^{1}([0, l] ; E)$ is said to be decomposable if for every $f, g \in M$ and each measurable subset $\theta$ in $[0, l]$,

$$
f \circ \chi_{\theta}+g \circ \chi_{[0, l] \backslash \theta} \in M
$$

where $\chi$ denotes the indicator of corresponding set.

See more details of this definition in $[2,6]$.

Theorem 2.9 (Bressan and Colombo). Let $(\Omega, d)$ be a separable metric space, let $X$ be a Banach space, and let $(J, \mathcal{A}, \mu)$ be a measure space (i.e., $\mathcal{A}$ is a $\sigma$-algebra on $J$ and $\mu$ is a measure on $(J, A)$ ) such that $\mu$ is nonatomic and $\mu(J)=1$. Consider the space $Y=L^{1}((J, \mathcal{A}, \mu), X)$ of integrable maps from $(J, \mathcal{A}, \mu)$ into $X$. If a multimap $F: \Omega \rightarrow Y$ is lsc with closed decomposable values, then $F$ has a continuous selection.

This statement is proved, for example, as [2, Lemma 9.2].

Now, we turn back to differential inclusion (1.2). Consider the manifold $M$ as above.

Definition 2.10. A set-valued vector field $F$ on $M$ is a set-valued map $F: M \rightarrow$ $T M$ such that $\pi \circ F=\mathrm{id}$, that is, $\pi(F(m))=m$ for each $m \in M$.

Definition 2.10 is a natural generalization of the standard definition of vector field to set-valued case.

Obviously, $F(t, m, X)$ in the right-hand side of (1.2) is a set-valued vector field on $M$ depending at each $m \in M$ on time $t$ and velocity $X \in T_{m} M$. Thus, it can be presented as a map $F: \mathbb{R} \times T M \rightarrow T M$ such that $\pi F(t, m, X)=m$ for all $t \in \mathbb{R}$ and $(m, X) \in T M$.

Definition 2.11. We say that the above-mentioned set-valued vector field $F(t, m$, $X)$ is lsc if it is lsc jointly in $(t, m, X)$ as a set-valued map $F: \mathbb{R} \times T M \rightarrow T M$. 


\section{The two-point boundary value problem}

For the sake of simplicity here, we suppose that the set-valued vector field $F(t$, $m, X)$ is defined for $t$ from a certain finite interval $I=[0, l] \subset \mathbb{R}$.

Introduce the norm $\|F(t, m, X)\|$ by standard formula

$$
\|F(t, m, X)\|=\sup _{y \in F(t, m, X)}\|y\| .
$$

Theorem 3.1. Let a point $m_{1} \in M$ be not conjugate with the point $m_{0} \in M$ along some geodesic $a(t)$ of the metric $\langle$,$\rangle and let the set-valued vector field F(t, m, X)$ with closed images be lsc and uniformly bounded, that is, $\|F(t, m, X)\|<k$ for a certain $k>0$ and for all $t, m, X$. There exists a number $L\left(m_{0}, m_{1}, a\right)$ such that for any $t_{0}, 0<t_{0}<L\left(m_{0}, m_{1}, a\right)$, inclusion (1.2) has a solution $m(t)$ such that $m(0)=$ $m_{0}$ and $m\left(t_{0}\right)=m_{1}$.

Proof. Let $I=[0, l]$. Consider the set-valued vector field $F(t, m(t), \dot{m}(t))$ defined along the $C^{1}$-curve $m(t)=\mathscr{S}(v(t)), v \in C^{0}\left(I, T_{m_{0}} M\right)$, and apply the parallel translation along $m(\cdot)$ at the point $m_{0}=m(0)$ to all the sets $F(t, m(t), \dot{m}(t))$. Then for any given $v$, we obtain the set-valued mapping of $\Gamma F(t, \mathscr{Y}(v(\cdot))$, $(d / d t) \mathscr{S}(v(\cdot)))$ from the segment $I$ to $T_{m_{0}} M$.

Lemma 3.2. The set-valued mapping

$$
\Gamma F\left(t, \mathscr{S}(v(\cdot)), \frac{d}{d t} \mathscr{S}(v(\cdot))\right): C^{0}\left(I, T_{m_{0}} M\right) \times I \longrightarrow T_{m_{0}} M
$$

is $l s c$.

Proof. Since $F(t, m, X)$ is lsc, the multimap $F(t, \mathscr{S}(v(t)),(d / d t) \mathscr{S}(v(t)))$ with values in $T M$ is lsc in $v$ as the operator $\mathscr{S}: C^{0}\left(I, T_{m_{0}} M\right) \rightarrow C_{m_{0}}^{1}(I, M)$ is a homeomorphism. Now applying the operator $\Gamma, \Gamma F(t, \mathscr{Y}(v(t)),(d / d t) \mathscr{S}(v(t)))$ is lsc since $\Gamma$ is continuous.

For any given $v$, denote by

$$
P \Gamma F\left(t, S(v(t)), \frac{d}{d t} S(v(t))\right)=\left\{y(\cdot): y(t) \in \Gamma F\left(t, S(v(t)), \frac{d}{d t} S(v(t))\right)\right\}
$$

the set of all measurable selections of the set-valued mapping

$$
\Gamma F\left(t, S(v(\cdot)), \frac{d}{d t} S(v(\cdot))\right): I \longrightarrow T_{m_{0}} M .
$$

Since the field $F$ is bounded by $k$ and the parallel translation preserves the norm of vectors for all $v$, the curves belonging to $P \Gamma F(t, S(v(t)),(d / d t) S(v(t)))$ are also bounded by the same $k$, that is, they are integrable. Thus, the mapping sending $v \in C^{0}\left(I, T_{m_{0}} M\right)$ to $P \Gamma F(t, S(v(t)),(d / d t) S(v(t)))$ is a multimap from 
$C^{0}\left(I, T_{m_{0}} M\right)$ to $L^{1}\left((I, \mathcal{A}, \mu), T_{m_{0}} M\right)$, where $\mathscr{A}$ is Borel $\sigma$-algebra and $\mu$ is the normalized Lebesgue measure. We can easily show that this multimap is lsc and that its values are decomposable. Thus from Theorem 2.9, it follows that it has a continuous selection, denote this selection by $p \Gamma F(t, S(v(\cdot)),(d / d t) S(v(\cdot)))$.

Clearly, a sufficiently small $t_{1}>0$ satisfies the inequality $t_{1}<L\left(m_{0}, m_{1}, k t_{1}, a\right)$ where $L\left(m_{0}, m_{1}, k t_{1}, a\right)$ is the number appearing in Theorem 2.3. We define the number $L\left(m_{0}, m_{1}, a\right)$ as the supremum of $t_{1}$ such that $t_{1}<L\left(m_{0}, m_{1}, k t_{1}, a\right)$.

Let $t_{0}<L\left(m_{0}, m_{1}, a\right)$. Without loss of generality, we can suppose that $I=\left[0, t_{0}\right]$. Consider the single-valued map

$$
B: C^{0}\left(\left[0, t_{0}\right], T_{m_{0}} M\right) \longrightarrow C^{0}\left(\left[0, t_{0}\right], T_{m_{0}} M\right)
$$

defined by the formula

$$
B v=\int_{0}^{t} p \Gamma F\left(s, S\left(v(\cdot)+C_{v}\right), \frac{d}{d t} S\left(v(\cdot)+C_{v}\right)\right) d s,
$$

where $C_{v}$ is the vector from Theorem 2.3.

Lemma 3.3. The map $B: C^{0}\left(I, T_{m_{0}} M\right) \rightarrow C^{0}\left(I, T_{m_{0}} M\right)$ is completely continuous.

Proof. By the construction for all $v$ and $t$, the sets $\Gamma F\left(t, \mathscr{S}\left(v(\cdot)+C_{v}\right),(d / d t) \mathscr{S}(v(\cdot)\right.$ $\left.+C_{v}\right)$ ) are bounded in $T_{m_{0}} M$ by the universal constant $k$. Hence, all selections $P \Gamma F\left(t, S\left(v(t)+C_{v}\right),(d / d t) S\left(v(t)+C_{v}\right)\right)$, in particular, all $p \Gamma F\left(t, S\left(v(\cdot)+C_{v}\right)\right.$, $\left.(d / d t) S\left(v(\cdot)+C_{v}\right)\right)$, are bounded by the same constant. This means that all curves

$$
\int_{0}^{t} p \Gamma F\left(s, \mathscr{Y}\left(v(\cdot)+C_{v}\right), \frac{d}{d s} S\left(v(\cdot)+C_{v}\right)\right) d s \in C^{0}\left(I, T_{m_{0}} M\right)
$$

are uniformly bounded and equicontinuous. Hence, $B\left(C^{0}\left(I, T_{m_{0}} M\right)\right)$ is compact in $C^{0}\left(I, T_{m_{0}} M\right)$.

By Theorem 2.9, $B: C^{0}\left(I, T_{m_{0}} M\right) \rightarrow L^{1}\left((I, \mathcal{A}, \mu), T_{m_{0}} M\right)$ is continuous. Since $C_{v}$ continuously depends on $v$ (see Theorem 2.3 ), this means that the vector

$$
\int_{0}^{l} p \Gamma F\left(s, \mathscr{Y}\left(v(\cdot)+C_{v}\right), \frac{d}{d s} S\left(v(\cdot)+C_{v}\right)\right) d s \in T_{m_{0}} M
$$

is continuous in $v \in C^{0}\left(I, T_{m_{0}} M\right)$. A very simple modification of the above arguments show that for a specified $t^{*} \in I$, the map sending $v(\cdot) \in C^{0}\left(I, T_{m_{0}} M\right)$ to the restriction of $p \Gamma F\left(t, S\left(v(\cdot)+C_{v}\right),(d / d t) S\left(v(\cdot)+C_{v}\right)\right)$ on $\left[0, t^{*}\right]$ is continuous as a map from $C^{0}\left(I, T_{m_{0}} M\right)$ to $L^{1}\left(\left(\left[0, t^{*}\right], \mathscr{A}, \mu\right), T_{m_{0}} M\right)$, hence we obtain that the vector $\int_{0}^{t^{*}} p \Gamma F\left(s, \mathscr{S}\left(v(\cdot)+C_{v}\right),(d / d s) S\left(v(\cdot)+C_{v}\right)\right) d s$ is continuous jointly in $t$ and $v$ for any specified $t^{*} \in I$. 
Thus for any $\varepsilon>0, v \in C^{0}\left(I, T_{m_{0}} M\right)$, and $t^{*} \in I$, there exists $\delta=\delta\left(\varepsilon, v, t^{*}\right)>0$ such that if $\left\|v(\cdot)-v_{1}(\cdot)\right\|_{C^{0}\left(I, T_{m_{0}} M\right)}<(1 / 2) \delta$ and $\left|t-t^{\prime}\right|<(1 / 2) \delta$,

$$
\begin{aligned}
& \| \int_{0}^{t} p \Gamma F\left(s, \mathscr{S}(v(\cdot)), \frac{d}{d s} S(v(\cdot))\right) d s \\
& \quad-\int_{0}^{t^{\prime}} p \Gamma F\left(s, \mathscr{S}\left(v_{1}(\cdot)\right), \frac{d}{d s} S\left(v_{1}(\cdot)\right)\right) d s \|_{T_{m_{0} M}}<\varepsilon .
\end{aligned}
$$

Since $I$ is compact, for given $v$, we can find unique $\delta=\delta(\varepsilon, v)$ for all $t \in I$. This completes the proof of continuity of $B: C^{0}\left(I, T_{m_{0}} M\right) \rightarrow C^{0}\left(I, T_{m_{0}} M\right)$.

Denote by $U_{k t_{0}}$ the ball in $C^{0}\left(\left[0, t_{0}\right], T_{m_{0}} M\right)$ with radius $k t_{0}$ centered at the origin. Since parallel translation preserves the norm of a vector, we can easily see that $B$ maps $U_{k t_{0}}$ into itself and therefore it has a fixed point $v_{0}(\cdot)$ in $U_{k t_{0}}$, that is, $v_{0}(\cdot)=B v_{0}(\cdot)$.

Taking into account (3.6), we can see that the equation $v_{0}(\cdot)=B v_{0}(\cdot)$ is a certain analogue of velocity hodograph equation (2.2). Thus, now we should demonstrate that $m(t)=S\left(v_{0}(t)+C_{v_{0}}\right)$ is the solution in question for (1.2). By construction, $m(0)=m_{0}, m\left(t_{0}\right)=m_{1}, m(t)$ is a $C^{1}$-curve, and $\dot{m}(t)$ is absolutely continuous. Since $v_{0}(\cdot)$ is a fixed point of $B$,

$$
\dot{v}_{0}(t)=p \Gamma F\left(t, S\left(v_{0}(t)+C_{v_{0}}\right), \frac{d}{d t} S\left(v_{0}(t)+C_{v_{0}}\right)\right)
$$

and from the definition of $p \Gamma F\left(t, S\left(v_{0}(t)+C_{v_{0}}\right),(d / d t) S\left(v_{0}(t)+C_{v_{0}}\right)\right)$, it follows that a.e.

$$
\dot{v}_{0}(t) \in \Gamma F\left(t, S\left(v_{0}(t)+C_{v_{0}}\right), \frac{d}{d t} S\left(v_{0}(t)+C_{v_{0}}\right)\right)
$$

Taking into account the properties of covariant derivative and the definition of operator $\Gamma$, and after parallel translation of $\dot{v}_{0}(t)$ and $\Gamma F\left(t, S\left(v_{0}(t)+C_{v_{0}}\right)\right.$, $\left.(d / d t) S\left(v_{0}(t)+C_{v_{0}}\right)\right)$ along $m(\cdot)$ to the point $m(t)$, we obtain $(D / d t) \dot{m}(t)$ and $F(t, m(t), \dot{m}(t))$, respectively. Thus, $(D / d t) \dot{m}(t) \in F(t, m(t), \dot{m}(t))$. This proves Theorem 3.1.

Consider a set-valued bounded and Hausdorff continuous force $A(t, m, X)$ with convex closed images.

Definition 3.4. A point $a$ in convex closed set $A$ is called extreme if there does not exist an open interval of straight line in $A$ that includes $a$. The multimap $\operatorname{Ext} A(t, m, X)$ is the set-valued map whose image at $(t, m, X)$ consists of extreme points of $A(t, m, X)$.

Lemma 3.5. For the set-valued force $A(t, m, X)$ mentioned above, the multimap $\operatorname{Ext} A(t, m, X)$ is $l s c$. 
Lemma 3.5 is a well-known fact of set-valued analysis. See, for example, [7, Lemma 2.1.1] and [2, Proposition 6.2]. Notice that $\operatorname{Ext} A(t, m, X)$ is bounded and definitely may not have convex images.

Consider a bounded Hausdorff continuous force field $A(t, m, X)$ with convex closed images on $M$ as above. We say that a trajectory $m(t)$ of the mechanical system with force $A(t, m, X)$ is governed by extreme values of controlling force if a.e. $(D / d t) \dot{m}(t)$ belongs to Ext $A(t, m(t), \dot{m}(t))$ (see Definition 3.4).

Theorem 3.6. If $m_{1}$ is not conjugate to $m_{0}$ along at least one geodesic, there exists a trajectory of the mechanical system with force $A(t, m, X)$, joining $m_{0}$ and $m_{1}$, that is governed by extreme values of the controlling force.

Proof. Consider the differential inclusion

$$
\frac{D}{d t} \dot{m}(t) \in \operatorname{Ext} A(t, m(t), \dot{m}(t))
$$

From boundedness of $A(t, m, X)$, it follows that $\operatorname{Ext} A(t, m, X)$ is bounded. Since, by Lemma 3.5, Ext $A(t, m(t), \dot{m}(t))$ is lsc, Theorem 3.6 follows from Theorem 3.1.

Theorem 3.6 is a criterion for controllability under the extreme values of controlling force.

Theorem 3.1 can be subjected to a certain generalization.

Definition 3.7. Let $I=[0, l] \subset \mathbb{R}$. The set-valued force field $F: I \times T M \rightarrow T M$ is called almost lsc if there exists a countable sequence of disjoint compact sets $\left\{I_{n}\right\}, I_{n} \subset I$ such that (i) the measure of $I \backslash \cup_{n} I_{n}$ is equal to zero and (ii) the restriction of $F$ on each $I_{n} \times T M$ is lsc.

Now consider (1.2) where $F$ is almost lsc and bounded.

COROLlary 3.8. The assertion of Theorem 3.1 remains true for $F$ almost lsc and bounded.

Corollary 3.8 follows from the fact that the set of measurable selections

$$
P \Gamma F\left(t, S(v(t)), \frac{d}{d t} S(v(t))\right)=\left\{y: y(t) \in \Gamma F\left(t, S(v(t)), \frac{d}{d t} S(v(t))\right)\right\}
$$

for almost lsc $F$ is lsc (see details in [6]).

Remark 3.9. If $M$ is a Euclidean linear space, any couple of points $m_{0} \neq m_{1}$ are not conjugate along the straight line joining them and we can easily see that the number $L\left(m_{0}, m_{1}, k, a\right)>0$ from Theorem 2.3 is equal to $\infty$ (see, e.g., $[3,5]$ for details). Hence the number $L\left(m_{0}, m_{1}, a\right)$ from Theorem 3.1 is also equal to $\infty$, and so Theorem 3.1 and Corollary 3.8 are valid for any couple of points $m_{0} \neq m_{1}$ and any $0<t_{0}<\infty$. 


\section{Acknowledgment}

The research is supported in part by Grant UR.04.01.008 of the Program "Universities of Russia” and by US CRDF-RF Ministry of Education Award VZ-010-0.

\section{References}

[1] R. L. Bishop and R. J. Crittenden, Geometry of Manifolds, Pure and Applied Mathematics, vol. 15, Academic Press, New York, 1964.

[2] K. Deimling, Multivalued Differential Equations, de Gruyter Series in Nonlinear Analysis and Applications, vol. 1, Walter de Gruyter, Berlin, 1992.

[3] Yu. E. Gliklikh, Riemannian parallel translation in nonlinear mechanics, Global Analysis-Studies and Applications I, Lect. Notes Math., vol. 1108, SpringerVerlag, 1984, pp. 128-151.

[4] _ Ordinary and Stochastic Differential Geometry as a Tool for Mathematical Physics, Mathematics and Its Applications, vol. 374, Kluwer Academic Publishers Group, Dordrecht, 1996.

[5] _ Global Analysis in Mathematical Physics. Geometric and Stochastic Methods, Applied Mathematical Sciences, vol. 122, Springer-Verlag, New York, 1997.

[6] M. Kamenskii, V. Obukhovskii, and P. Zecca, Condensing Multivalued Maps and Semilinear Differential Inclusions in Banach Spaces, de Gruyter Series in Nonlinear Analysis and Applications, vol. 7, Walter de Gruyter, Berlin, 2001.

[7] A. A. Tolstonogov, Differential Inclusions in a Banach Space, "Nauka" Sibirsk. Otdel., Novosibirsk, 1986 (Russian).

Yuri E. Gliklikh: The Faculty of Mathematics, Voronezh State University, 1 University Square, 394006 Voronezh, Russia

E-mail address: yuri@yeg.vsu.ru

Andrei V. Obukhovskiĭ: The Faculty of Mathematics, Voronezh State University, 1 University Square, 394006 Voronezh, Russia

E-mail address: avo@mathd.vsu.ru 


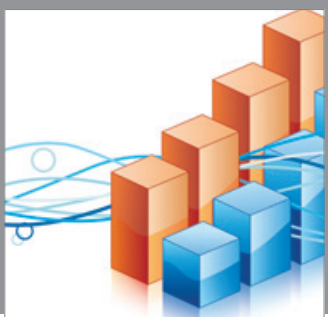

Advances in

Operations Research

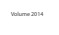

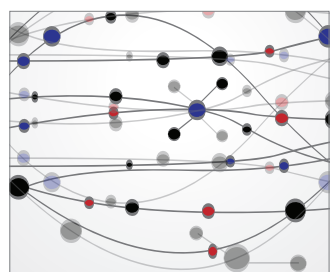

\section{The Scientific} World Journal
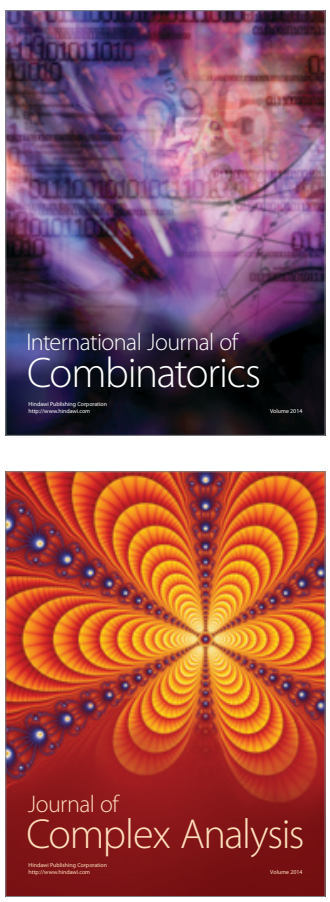

International Journal of

Mathematics and

Mathematical

Sciences
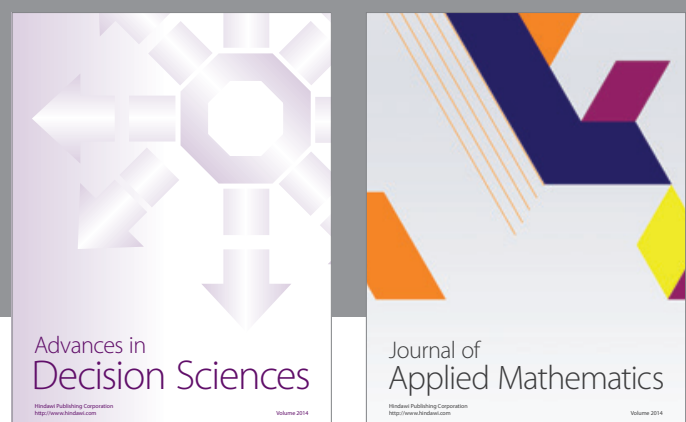

Journal of

Applied Mathematics
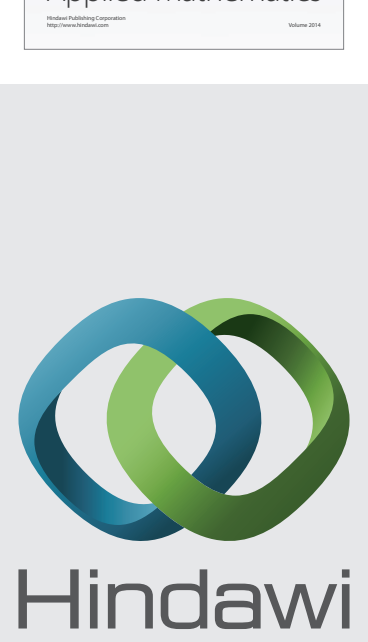

Submit your manuscripts at http://www.hindawi.com
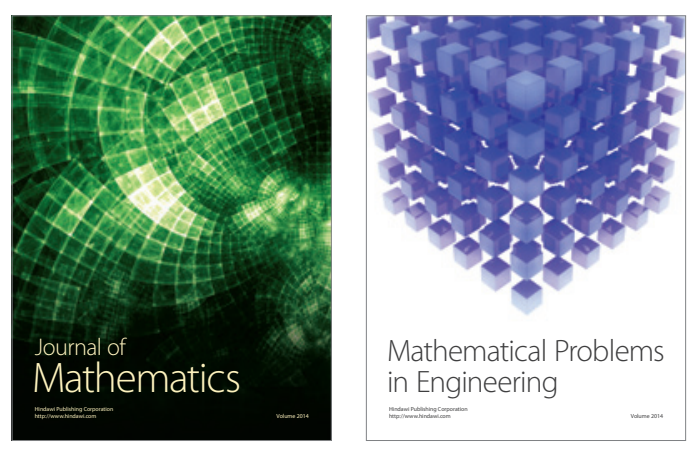

Mathematical Problems in Engineering
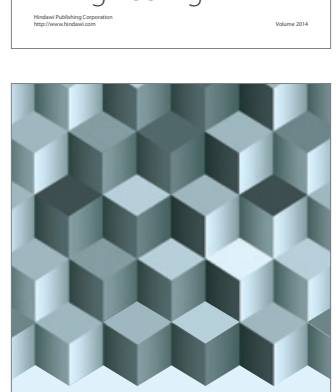

Journal of

Function Spaces
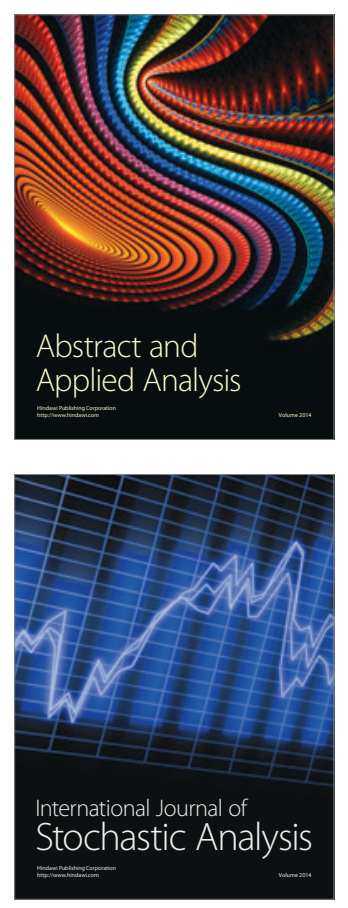

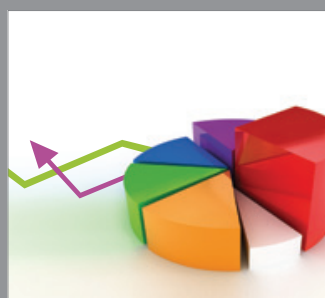

ournal of

Probability and Statistics

Promensencen
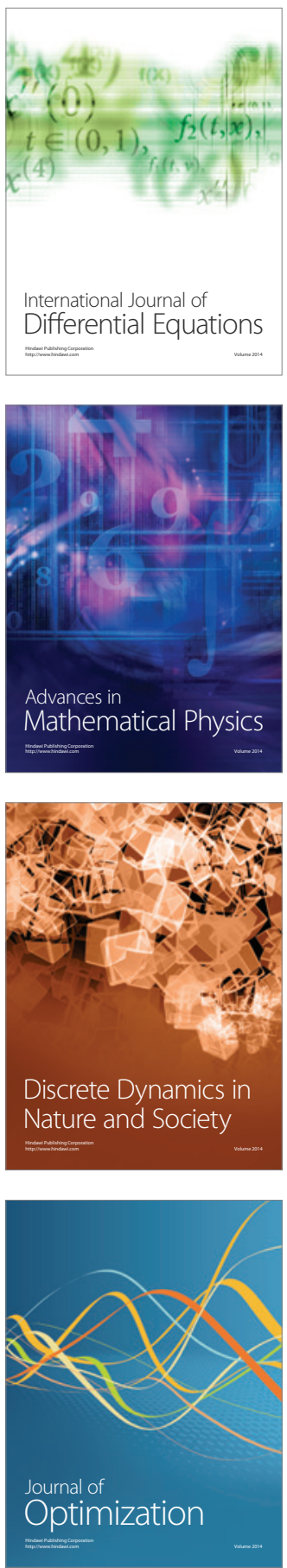